\title{
On torus knots and unknots
}

\author{
Chiara Oberti and Renzo L. Ricca* \\ Department of Mathematics and Applications, \\ University of Milano-Bicocca, \\ Via Cozzi 55, 20125 Milano, Italy \\ *renzo.ricca@unimib.it
}

Received 5 April 2015

Accepted 31 March 2016

Published 28 April 2016

\begin{abstract}
A comprehensive study of geometric and topological properties of torus knots and unknots is presented. Torus knots/unknots are particularly symmetric, closed, space curves, that wrap the surface of a mathematical torus a number of times in the longitudinal and meridian direction. By using a standard parametrization, new results on local and global properties are found. In particular, we demonstrate the existence of inflection points for a given critical aspect ratio, determine the location and prescribe the regularization condition to remove the local singularity associated with torsion. Since to first approximation total length grows linearly with the number of coils, its nondimensional counterpart is proportional to the topological crossing number of the knot type. We analyze several global geometric quantities, such as total curvature, writhing number, total torsion, and geometric 'energies' given by total squared curvature and torsion, in relation to knot complexity measured by the winding number. We conclude with a brief presentation of research topics, where geometric and topological information on torus knots/unknots finds useful application.
\end{abstract}

Keywords: Torus knots; parametric equations; curvature; torsion; linking number; writhe.

Mathematics Subject Classification 2010: 57M25, 57M27, 78A25

\section{Introduction}

Torus knots and unknots are particularly simple, closed, space curves that by definition wrap the surface of a mathematical torus in the longitudinal and meridian direction, forming highly symmetric configurations (see Fig. 1). In this case, they provide a rare example of nontrivial, closed, space curves, whose geometric and topological properties can be easily investigated by analytical means. For these reasons, torus knots and unknots represent an interesting case study where relations between geometry and topology can be explored accurately. This makes them a

\footnotetext{
* Corresponding author.
} 

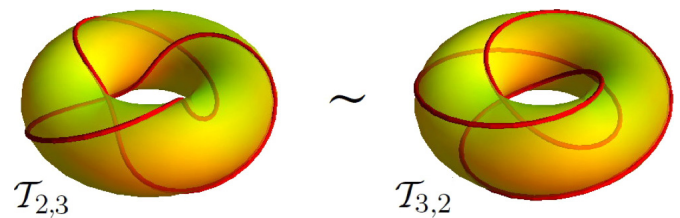

(a)
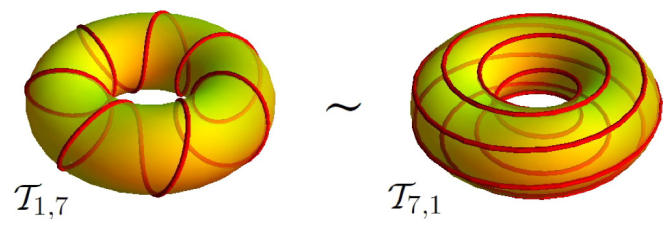

(b)

Fig. 1. (Color online) Torus knots/unknot (red online) on the mathematical torus. (a) Standard trefoil knot $\mathcal{T}_{2,3}$ and torus knot $\mathcal{T}_{3,2}$ topologically equivalent to $\mathcal{T}_{2,3}$. (b) Poloidal coil $\mathcal{T}_{1,7}$ and toroidal coil $\mathcal{T}_{7,1}$, both topologically equivalent to the standard unknot (i.e. the standard circle).

privileged object in a broad spectrum of disciplines, from pure mathematics to physics, biology and engineering, where aspects of structural complexity are important in their own right, or in relation to function.

Elastic filaments in the shape of torus knots have been studied in relation to bending and torsional energy [1], in terms of Möbius 'energy' [2], and to understand packing and structural complexity properties of ideal knots [3] and polymers $[4,5]$. As solutions to differential equations, torus knots have been found in the theory of integrable systems [6], in Maxwell's theory of electromagnetism [7], as phase singularities in optics [8, 9], and even as Wilson loops in Chern-Simons topological quantum field theory $[10,11]$. In fluid flows, torus knots and unknots have been studied in the context of classical and superfluid vortex dynamics $[12,13]$, and they may arise as Bose-Einstein condensates of ultracold atoms [14], as colloids [15] and in many other physical, chemical and biological contexts.

A systematic, comprehensive study of their geometric and topological properties, however, seems to be missing in literature. With the present work, we want to fill this gap by presenting a collection of results (mainly geometric in character) of interest in pure and applied mathematics. A formal definition of torus knots and unknots is given in Sec. 2, by introducing standard parametrization, and symmetry aspects. A detailed analysis of local properties is presented in Sec. 3, by examining curvature, inflection points and torsion. For every torus knot/unknot, we determine the conditions for the existence and location of inflection points (Theorem 3.2) and prescribe the regularity conditions necessary to remove the local singularity associated with torsion (Theorem 3). Global properties are discussed in Sec. 4. Since to a first approximation total length grows linearly with the number of coils we show that its nondimensional counterpart is proportional to the topological crossing 
number. We analyze several global geometric quantities, such as total curvature, writhing number, total torsion, total squared curvature and torsion (where the latter two are related to bending and torsional energy of elastic rods) in terms of knot complexity given by the winding number. By using information on the tangent indicatrix, we show how the development of inflectional configurations contributes to the growth of the writhing number and the jump of intrinsic twist in the framed knot. In the last section, we conclude with a brief discussion of possible applications in active areas of current research.

\section{Torus Knots and Unknots: Standard Parametrization and Symmetry Aspects}

Torus knots and unknots can be represented by particularly symmetric, closed curves, that lie on the surface of a mathematical torus, wrapped uniformly around it. A torus knot/unknot $\mathcal{T}_{p, q}$ wraps the torus $p$ times along the longitudinal (or toroidal) direction, and $q$ times along the meridian (or poloidal) direction $[16$, pp. 136-141]. Torus knots are given by taking $p>1$ and $q>1$, with $\{p, q\}$ co-prime integers (see Fig. 1(a)). If either $p=1$ or $q=1$ we have multiply coiled curves (see Fig. 1(b)) topologically equivalent to the unknot, i.e. the standard circle. The ratio $w=q / p(w>0)$ is the winding number of $\mathcal{T}_{p, q}$, and provides a measure of geometric complexity of the knot/unknot. Note that for any $p$ finite and fixed, if $q \rightarrow \infty$ the curve tends to cover the mathematical torus in the poloidal direction with infinitely many turns to form a poloidal hollow ring. Similarly, for any $q$ finite and fixed, if $p \rightarrow \infty$ the curve covers the torus with infinitely many turns in the toroidal direction forming a toroidal hollow ring.

In general for given $p$ and $q$ the two knots $\mathcal{T}_{p, q}$ and $\mathcal{T}_{q, p}$ are topologically equivalent, i.e. one can be transformed into the other by continuous deformation; hence $\mathcal{T}_{p, q} \sim \mathcal{T}_{q, p}$. A standard measure of topological complexity of knots is provided by the minimum crossing number $c_{\text {min }}$, that for torus knots [17] is given by

$$
c_{\min }\left(\mathcal{T}_{p, q}\right)=\min [p(q-1), q(p-1)] .
$$

Since $c_{\min }$ is a topological invariant of torus knots, for given $p$ and $q$ we have $c_{\min }\left(\mathcal{T}_{p, q}\right)=c_{\min }\left(\mathcal{T}_{q, p}\right)$. If $q>p$, then $q(p-1)<p(q-1)$ and $c_{\min }=q(p-1)$ (alternatively if $p>q$, then $c_{\min }=p(q-1)$ ).

The standard parametrization of a torus knot/unknot $\mathcal{T}_{p, q}$ of winding number $w$ is given by

$$
\mathbf{X}=\mathbf{X}(\alpha):\left\{\begin{array}{l}
x=(R+r \cos w \alpha) \cos \alpha \\
y=(R+r \cos w \alpha) \sin \alpha \\
z=r \sin w \alpha
\end{array}\right.
$$

where $\mathbf{X}=(x(\alpha), y(\alpha), z(\alpha))$ denotes the vector position of a point on $\mathcal{T}_{p, q}$ and $\alpha \in[0,2 \pi p)$ is a parameter on the curve; $R$ and $r$ are respectively the toroidal and 
poloidal radius of the torus, with $0<r<R$ (for $r=0$ the torus collapses to the standard circle of radius $R$, and for $r \geq R$ the torus develops self-intersections). Any prescription $r=r(\alpha, \beta)$ smooth function of the toroidal and poloidal angles $\alpha$ and $\beta=w \alpha$ gives rise to more general toroidal curves with periodic functional dependence on both directions, more general knot presentations of same knot type. The geometric features discussed below are therefore qualitatively preserved under composition of continuous maps.

By introducing the aspect ratio $\lambda=r / R$ with $\lambda \in(0,1)$, Eqs. (2.2) become

$$
\mathbf{X}=\mathbf{X}(\alpha):\left\{\begin{array}{l}
x=R(1+\lambda \cos w \alpha) \cos \alpha, \\
y=R(1+\lambda \cos w \alpha) \sin \alpha, \\
z=R \lambda \sin w \alpha .
\end{array}\right.
$$

Every knot/unknot parametrized by Eqs. (2.3) is a smooth and simple curve (i.e $C^{\infty}$ without self-intersections) in $\mathbb{R}^{3}$.

Standard torus knots/unknots can be thought of as generated by the action of $(q-1)$ rigid rotations around the $z$-axis of the fundamental section of the curve given by $\alpha \in[0,2 \pi / w$ ) (see Fig. 2), with the fundamental sector in between the half-planes $\theta=0$ and $\theta=2 \pi / q$. The geometry of the curve is thus completely determined by the properties of the curve in the fundamental section of period $2 \pi / w$.
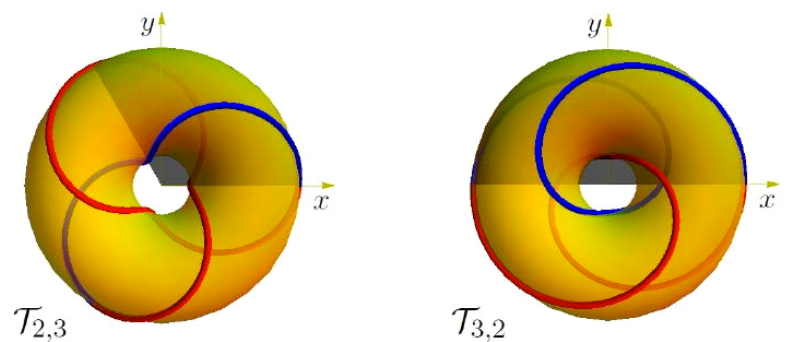

(a)
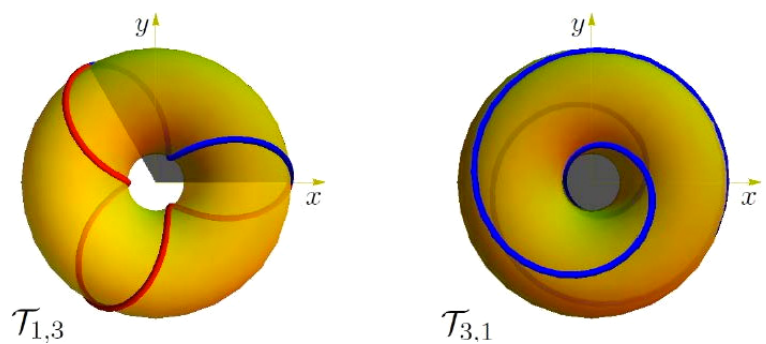

(b)

Fig. 2. (Color online) Knot fundamental section (blue curve online) and fundamental sector (darker region). (a) Knots $\mathcal{T}_{2,3}$ and $\mathcal{T}_{3,2}$. (b) Unknots $\mathcal{T}_{1,3}$ and $\mathcal{T}_{3,1}$. 


\section{Local Properties: Curvature, Inflection Points and Torsion}

\subsection{Curvature and inflection points}

In general, a smooth curve parametrized by $\mathbf{r}(t)(t \in[a, b] \subset \mathbb{R})$ is said to be regular if the tangent vector $\mathbf{r}^{\prime}(t)$ (where prime denotes differentiation) never vanishes for all values of $t$. By direct application of this definition one can prove the following:

Theorem 3.1. Every $\mathcal{T}_{p, q}$ parametrized by Eqs. (2.3) is a regular curve in $\mathbb{R}^{3}$.

A point where $\mathbf{r}^{\prime}(t)=0$ (for some $t \in[a, b]$ ) is said to be a singular point of order 0 ; if $\mathbf{r}^{\prime \prime}(t)=0$ with $\mathbf{r}^{\prime}(t) \neq 0$, the point is a singular point of order 1 , or an inflection point of the curve. From the standard definition [18] of curvature

$$
c(t)=\frac{\left|\mathbf{r}^{\prime}(t) \times \mathbf{r}^{\prime \prime}(t)\right|}{\left|\mathbf{r}^{\prime}(t)\right|^{3}},
$$

a point on a smooth and regular curve, where curvature $c(t)=0$ in isolation, is called an inflection point. The study of inflectional configurations is important in its own right, as well as for applications. Let us call $\lambda_{\mathrm{cr}}$ the critical aspect ratio for $\mathcal{T}_{p, q}$ in inflectional state (i.e. when $\mathcal{T}_{p, q}$ has at least one inflection point in isolation). We have:

Theorem 3.2. Every $\mathcal{T}_{p, q}$ of winding number $w$, parametrized by Eqs. (2.3), has at least $q$ points of inflection, placed at $\alpha=(2 k+1) \pi / w(k=0,1,2, \ldots, q-1)$, when $\lambda=\lambda_{\text {cr }}=\left(1+w^{2}\right)^{-1}$.

Proof. Let us consider $\lambda$ a kinematic parameter; Eqs. (2.3) describe a deformation process of the curve through an inflectional configuration. By using (2.3) and (3.1), from $c=0$ in the fundamental section we have $\alpha=\pi / w$. Since for every $\mathcal{T}_{p, q}$ there are exactly $q-1$ repeats over the whole curve we have at least $q$ inflection points in isolation for $\alpha \in[0,2 \pi p)$. In general, we have

$$
\alpha=\frac{\pi}{w}: \quad c=\frac{1-\lambda-\lambda w^{2}}{R(1-\lambda)^{2}+\lambda^{2} w^{2}} ;
$$

hence, from (3.2) and $c=0$, we have

$$
1-\lambda-\lambda w^{2}=0 \Leftrightarrow \lambda=\lambda_{\mathrm{cr}}=\frac{1}{1+w^{2}} .
$$

By using information on geodesic and normal components of curvature, Fuller [19, pp. 42-54] proved that $\mathcal{T}_{p, q}$ has exactly $q$ points of inflection in isolation, and that $\lambda=\lambda_{\text {cr }}$ is the only critical aspect ratio.

Curvature $c$, plotted in the fundamental section against $\alpha$, is shown in Fig. 3 for several values of $\lambda(R=1)$. Note that for $\lambda=\lambda_{\mathrm{cr}}$ the curvature vanishes and the inflection point is in the middle of the curvature period. For example the knot $\mathcal{T}_{2,3}$ has 3 inflection points at $\alpha=2 \pi / 3,3(2 \pi / 3)$ and $5(2 \pi / 3)$ when $\lambda_{\text {cr }}=4 / 13$. 

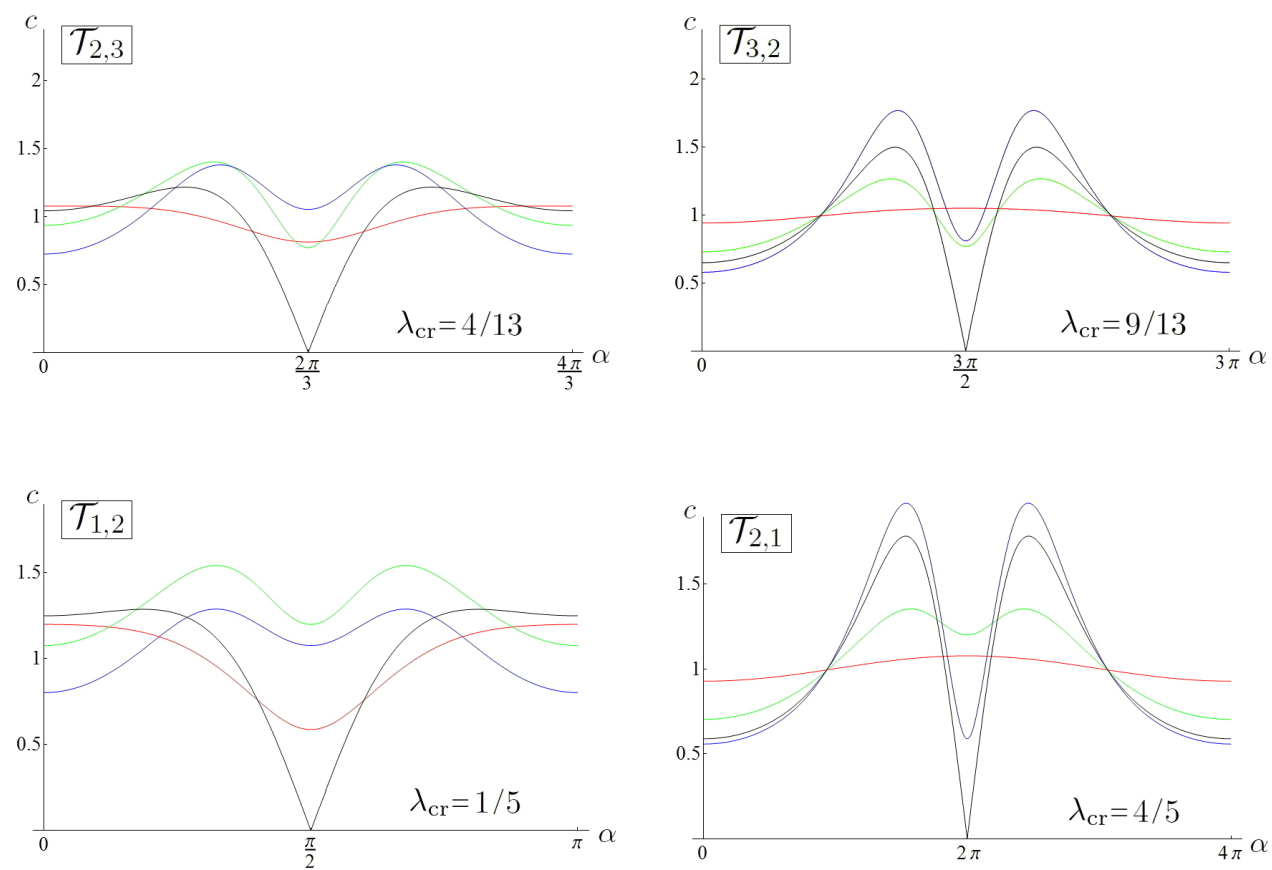

Fig. 3. (Color online) Curvature $c$ plotted against $\alpha$ in the fundamental section, for several values of $\lambda$ with $R=1$ (online: $\lambda=0.1$ red, $\lambda=0.5$ green, $\lambda=0.9$ blue and $\lambda=\lambda_{\text {cr }}$ black). Top row: knots $\mathcal{T}_{2,3}$ and $\mathcal{T}_{3,2}$; bottom row: unknots $\mathcal{T}_{1,2}$ and $\mathcal{T}_{2,1}$.

As $\lambda \rightarrow \lambda_{\mathrm{cr}}$, the curvature develops two maxima, a generic feature of all torus knots/unknots. When toroidal wraps dominate, $c \rightarrow 1 / R$ when $\lambda \rightarrow 0$ (i.e. when the torus collapses to the standard circle); when poloidal wraps dominate, $c \rightarrow 1 / \lambda$ when $\lambda \rightarrow 1$ (i.e. when the hole of the torus shrinks to a point).

\subsection{Torsion}

In general, torsion [18] is defined by

$$
\tau(t)=\frac{\left(\mathbf{r}^{\prime}(t) \times \mathbf{r}^{\prime \prime}(t)\right) \cdot \mathbf{r}^{\prime \prime \prime}(t)}{\left|\mathbf{r}^{\prime}(t) \times \mathbf{r}^{\prime \prime}(t)\right|^{2}} .
$$

At an inflection point torsion $\tau$ becomes singular, but integrable [20, 21]. For $\mathcal{T}_{p, q}$ the singularity is removable by continuity, by taking the limit from both sides along the curve. By taking Taylor's expansion of the numerator and denominator of $\tau$ near $\alpha=\pi / w$ (in the fundamental section), one can prove the following result.

Theorem 3.3. Let $\mathcal{T}_{p, q}$ be a torus knot/unknot parametrized by Eqs. (2.3) with $\lambda=\lambda_{\mathrm{cr}}$. The singularity of torsion at the inflection point is removable by continuity 

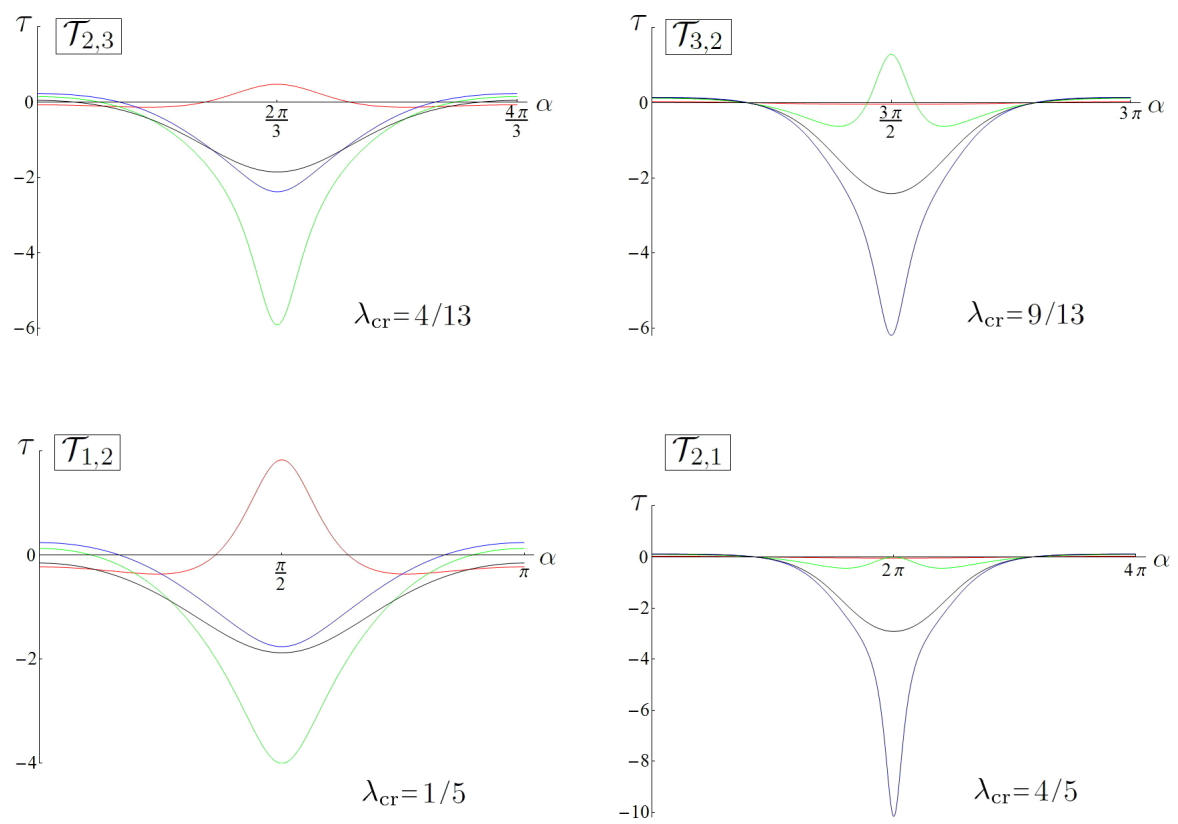

Fig. 4. (Color online) Torsion $\tau$ plotted against $\alpha$ in the fundamental section, for several values of $\lambda$ with $R=1$ (online: $\lambda=0.1$ red, $\lambda=0.5$ green, $\lambda=0.9$ blue and $\lambda=\lambda_{\mathrm{cr}}$ black). Top row: knots $\mathcal{T}_{2,3}$ and $\mathcal{T}_{3,2}$; bottom row: unknots $\mathcal{T}_{1,2}$ and $\mathcal{T}_{2,1}$. Torsion has been regularized by applying Eq. (3.5) for $\lambda=\lambda_{\mathrm{cr}}$.

by taking

$$
\alpha=\frac{\pi}{w}: \quad \tau=-\frac{\left(10+7 w^{2}+w^{4}\right)\left(1+w^{2}\right)}{2 R w\left(2+w^{2}\right)^{2}} .
$$

The diagrams of Fig. 4 show an application of Theorem 3.3, where the singularity of torsion has been removed by applying Eq. (3.5). From the limiting form of torsion at the point of inflection, we have

Corollary 3.4. The value of torsion at the inflection point never vanishes.

Several bounds on aspect ratio and winding number for nonvanishing torsion were found by Rodriguez Costa [22].

\section{Global Properties}

\subsection{Total length}

From standard definition and by using the parametrization (2.3) total length $L$ of $\mathcal{T}_{p, q}$ is given by

$$
L=\int_{0}^{2 \pi p}\left|\mathbf{X}^{\prime}(\alpha)\right| \mathrm{d} \alpha=R \int_{0}^{2 \pi p} \sqrt{(1+\lambda \cos w \alpha)^{2}+\lambda^{2} w^{2}} \mathrm{~d} \alpha .
$$


It is useful to consider nondimensional quantities: by normalizing $L$ with respect to $L_{0}=2 \pi R$ (length of the torus axis), we have

$$
\bar{L}=\frac{1}{2 \pi} \int_{0}^{2 \pi p} \sqrt{(1+\lambda \cos w \alpha)^{2}+\lambda^{2} w^{2}} \mathrm{~d} \alpha .
$$

The normalized total length is plotted in Fig. 5 (top-left diagram) against $w$, for several knots and unknots. The following result can be easily proven:

Theorem 4.1. Let $\mathcal{T}_{p, q}$ be a torus knot/unknot with $\bar{L}$ given by Eq. (4.2). We have:

(i) for given $p$, if $q \gg p$, then $\bar{L} \approx \lambda q$;

(ii) for given $q$, if $p \gg q$, then $\bar{L} \approx(1+\lambda) p$.

Since for $q \gg p, c_{\min }=q(p-1)$, by the first of (4.3) we have

$$
\bar{L} \approx \frac{\lambda}{p-1} c_{\min }
$$

Similarly, for $p \gg q$ :

$$
\bar{L} \approx \frac{1+\lambda}{q-1} c_{\min } .
$$

Lower and upper bounds for $\bar{L}$ can be readily found by using $|\cos w \alpha| \leq 1$ and by direct integration of (4.2) we have

$$
p \sqrt{(1-\lambda)^{2}+\lambda^{2} w^{2}} \leq \bar{L} \leq p \sqrt{(1+\lambda)^{2}+\lambda^{2} w^{2}}
$$

\subsection{Total curvature and writhing number}

The total curvature of $\mathcal{T}_{p, q}$ is given by

$$
K=\int_{0}^{2 \pi p} c(\alpha)\left|\mathbf{X}^{\prime}(\alpha)\right| \mathrm{d} \alpha
$$

a nondimensional quantity. The top-right diagram of Fig. 5 shows $K=K(w)$ for several torus knots/unknots considered. Since length grows linearly with the number of wraps, and each wrap contributes equally to $K$, a more informative quantity is the total curvature per unit length $\bar{K}$ (also a dimensionless quantity), given by

$$
\bar{K}=\frac{K}{\bar{L}} \text {. }
$$

Figure 5 shows the plot $\bar{K}=\bar{K}(w)$ for $\lambda=0.5, R=1$. As $w \rightarrow 0, \bar{K} \rightarrow 2 \pi$ (total curvature per unit length of the torus axis); as $w \rightarrow \infty, \bar{K} \rightarrow 2 \pi / \lambda$ (total curvature per unit length of the torus cross-sectional circle).

The writhing number $\mathrm{Wr}[23]$ of $\mathcal{T}_{p, q}$ is given by

$$
\mathrm{Wr}=\frac{1}{4 \pi} \int_{0}^{2 \pi p} \int_{0}^{2 \pi p} \frac{\left(\mathbf{X}^{\prime}(\alpha) \times \mathbf{X}^{\prime}\left(\alpha^{*}\right)\right) \cdot\left(\mathbf{X}(\alpha)-\mathbf{X}\left(\alpha^{*}\right)\right)}{\left|\mathbf{X}(\alpha)-\mathbf{X}\left(\alpha^{*}\right)\right|^{3}} \mathrm{~d} \alpha \mathrm{d} \alpha^{*},
$$

where $\mathbf{X}(\alpha)$ and $\mathbf{X}\left(\alpha^{*}\right)$ are two points on $\mathcal{T}_{p, q}$. The writhing number is a pure, real number of considerable importance in applications (see last section). Straightforward computation of (4.9) based on (2.3) gives negative values of $\mathrm{Wr}=\mathrm{Wr}(w)$ due 

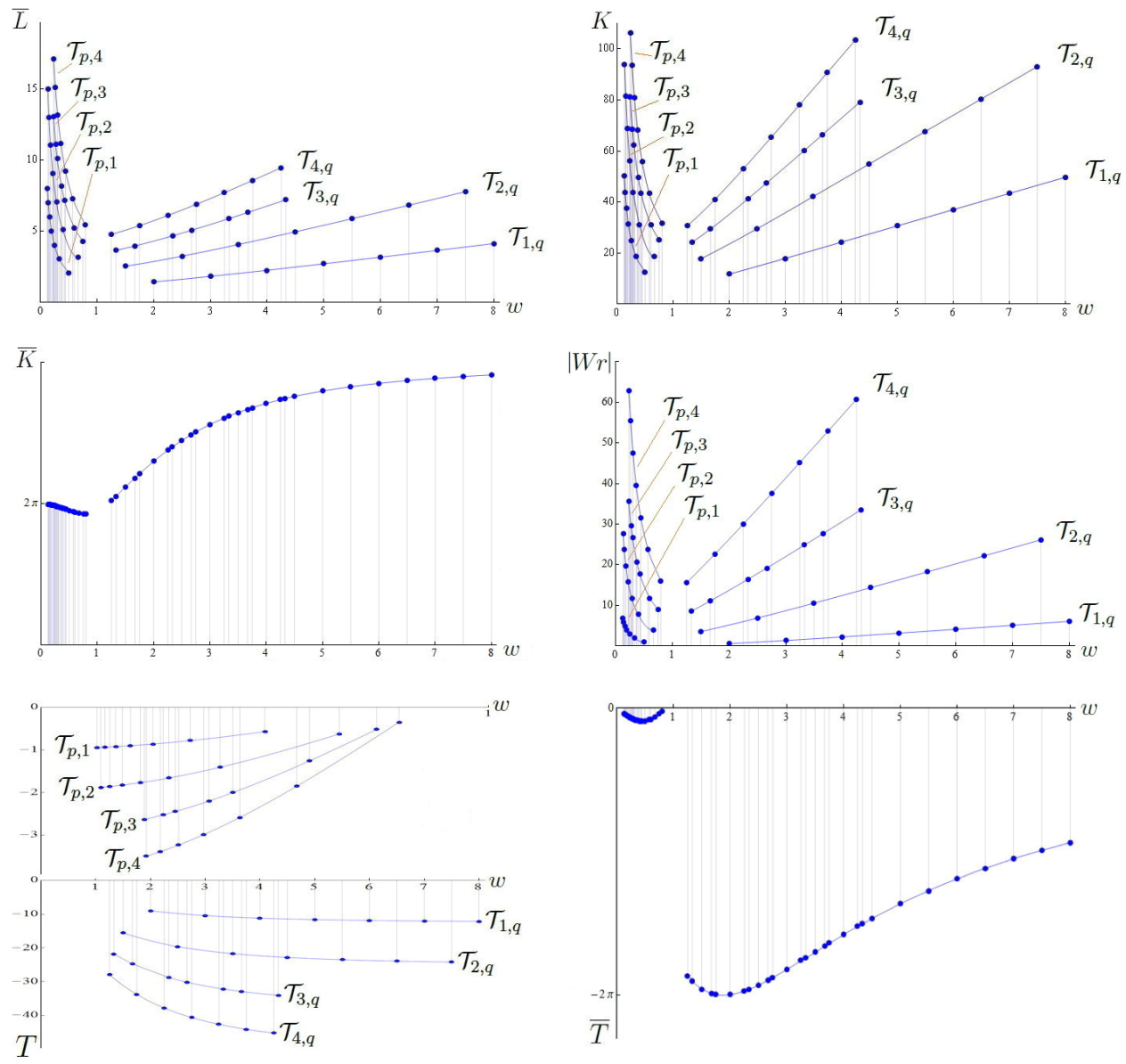

$$
\lambda=0.5
$$

Fig. 5. Top diagrams: normalized total length $\bar{L}$ and total curvature $K$; central diagrams: total curvature per unit length $\bar{K}$ and writhing number $|\mathrm{Wr}|$; bottom diagrams: total torsion $T$ and total torsion per unit length $\bar{T}$. All plots are for $\lambda=0.5$ and $R=1$ against $w: \mathcal{T}_{p, 1}$ and $\mathcal{T}_{1, q}, p, q=\{2,3,4,5,6,7,8\} ; \mathcal{T}_{p, 2}$ and $\mathcal{T}_{2, q}, p, q=\{3,5,7,9,11,13,15\} ; \mathcal{T}_{p, 3}$ and $\mathcal{T}_{3, q}$, $p, q=\{4,5,7,8,10,11,13\} ; \mathcal{T}_{p, 4}$ and $\mathcal{T}_{4, q}, p, q=\{5,7,9,11,13,15,17\}$. Interpolation is made for visualization purposes.

to the handedness associated with the parametrization chosen. Since total curvature and writhe are quantities closely related, for ease of comparison we plot $|\mathrm{Wr}(w)|$ instead (see Fig. 5, center-right diagram).

The presence of inflectional configurations has important consequences for writhe. The relation can be made explicit by mapping $\mathcal{T}_{p, q}$ to the tangent indicatrix (tantrix) $I_{\mathbf{t}}$ on the unit sphere by the tangent map $\mathbf{t}[24,25]$ (see Fig. 6). Let $A$ be the total area on the unit sphere (solid angle in steradians, counted with multiplicity [26]), enclosed by the tantrix curve (with its own self-intersections). The 

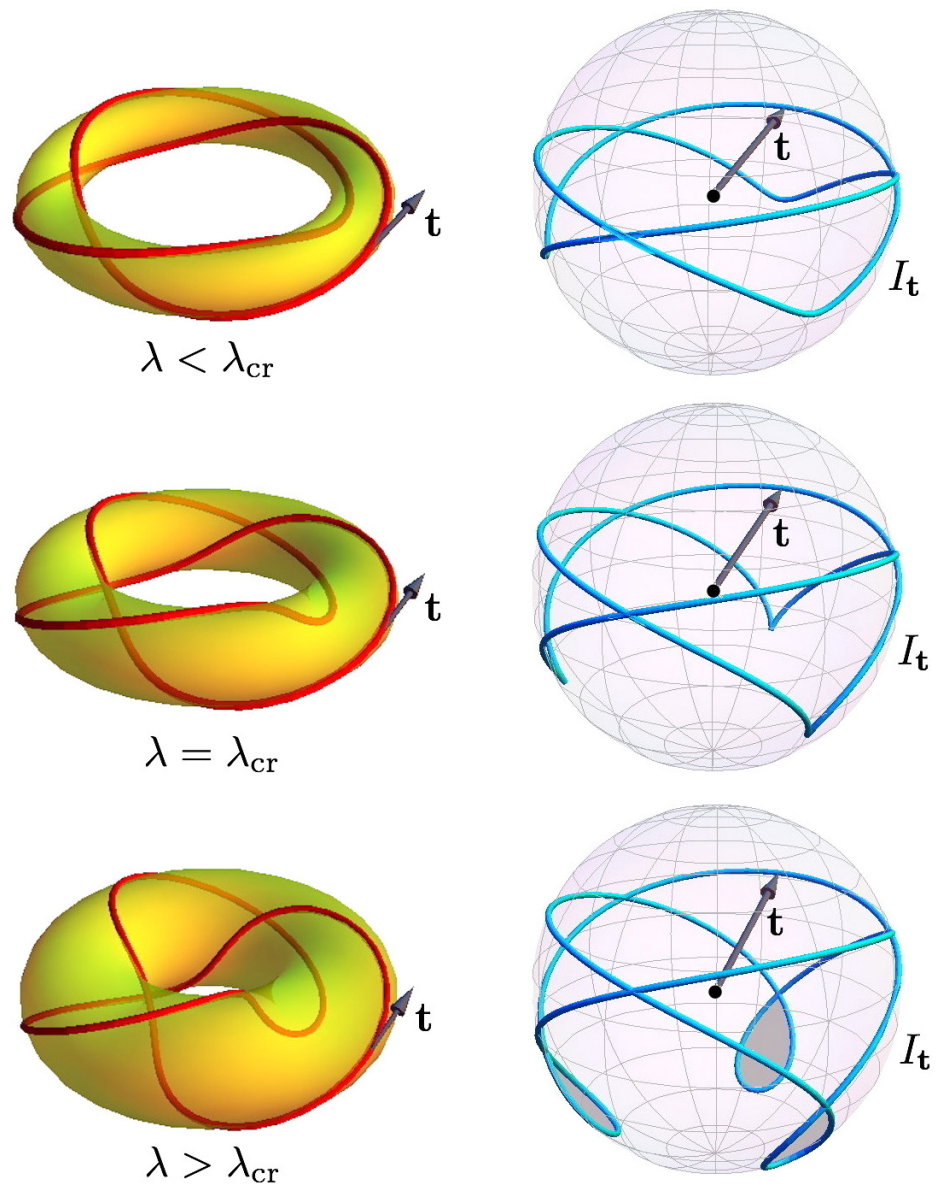

Fig. 6. (Color online) Knot $\mathcal{T}_{2,3}$ and tantrix $I_{\mathbf{t}}$ (on the right) for $\lambda=0.2<\lambda_{\mathrm{cr}}$ (top), $\lambda=4 / 13=$ $\lambda_{\text {cr }}$ (center) and $\lambda=0.5>\lambda_{\text {cr }}$ (bottom). Note the three cusps on the critical tantrix (center-right diagram); for $\lambda>\lambda_{\mathrm{cr}}$ a single loop develops from each cusp, contributing with additional spherical area (in grey) to total writhe.

writhing number of $\mathcal{T}_{p, q}$ admits interpretation in terms of spherical area, according to $[24]$

$$
\mathrm{Wr}=\frac{A}{2 \pi}+1 \quad(\bmod 2) .
$$

Since the unit tangent to the tantrix is the unit normal vector to $\mathcal{T}_{p, q}, q$ inflection points on $\mathcal{T}_{p, q}$ are mapped to $q$ cusps on the corresponding tantrix (see the center-right diagram of Fig. 6). By considering the deformation of the tantrix as $\lambda$ increases, we see the development of $q$ cusps (at $\lambda=\lambda_{\mathrm{cr}}$ ), each generating a single loop when $\lambda>\lambda_{\text {cr }}$; each loop contributes to the total area with an additional area given by the grey regions of Fig. 6 (bottom-right diagram), hence according to Eq. (4.10) to total writhe. 


\subsection{Total torsion}

The total torsion of $\mathcal{T}_{p, q}$ is given by

$$
T=\int_{0}^{2 \pi p} \tau(\alpha)\left|\mathbf{X}^{\prime}(\alpha)\right| \mathrm{d} \alpha .
$$

Since $T$ is the inverse of a radius of torsion (the second radius of curvature in $\mathbb{R}^{3}$ ), total torsion is also nondimensional. The bottom-left diagrams of Fig. 5 show $T=T(w)$ for $w<1$ and $w>1(\lambda=0.5)$. As expected, poloidal wraps tend to contribute more than toroidal wraps to total torsion (in absolute value).

Since each wrap contributes to total torsion equally, we introduce the total torsion per unit length $\bar{T}$, defined by

$$
\bar{T}=\frac{T}{\bar{L}}
$$

The plot of $\bar{T}=\bar{T}(w)$ shows that the values of all torus knots/unknots (within each family $w<1, w>1$ ) follow the same power law, represented by the single, dotted curve on the bottom-right diagram of Fig. 5.

\subsection{Total squared curvature and torsion}

Global functionals of curvature and torsion have been widely studied [27-30]. Particularly important are the integrals of the squared curvature and squared torsion, that are related to bending and torsional energy of elastic filaments (see [31]; also, for example, [1] and [32]). These are given by

$$
E_{b}=\int_{0}^{2 \pi p} c^{2}(\alpha)\left|\mathbf{X}^{\prime}(\alpha)\right| \mathrm{d} \alpha, \quad E_{\tau}=\int_{0}^{2 \pi p} \tau^{2}(\alpha)\left|\mathbf{X}^{\prime}(\alpha)\right| \mathrm{d} \alpha .
$$

Nondimensional quantities are obtained by normalizing $E_{b}$ and $E_{\tau}$ with respect to the total squared curvature of the unit circle $E_{0}=2 \pi$. These are given by

$$
\begin{aligned}
& \bar{E}_{b}=\frac{E_{b}}{E_{0}}=\frac{1}{2 \pi} \int_{0}^{2 \pi p} c^{2}(\alpha)\left|\mathbf{X}^{\prime}(\alpha)\right| \mathrm{d} \alpha, \\
& \bar{E}_{\tau}=\frac{E_{\tau}}{E_{0}}=\frac{1}{2 \pi} \int_{0}^{2 \pi p} \tau^{2}(\alpha)\left|\mathbf{X}^{\prime}(\alpha)\right| \mathrm{d} \alpha .
\end{aligned}
$$

Plots of $\bar{E}_{b}=\bar{E}_{b}(w)$ and $\bar{E}_{\tau}=\bar{E}_{\tau}(w)$ are shown in Fig. 7 for $\lambda=0.5$ and $R=1$. Note that contributions from torsional energy of $\mathcal{T}_{p, 1}-\mathcal{T}_{p, 4}$ are an order of magnitude smaller than those from bending energy. More generally, the relative contribution of toroidal/poloidal wraps to bending/torsional energy depends on the particular aspect ratio, so that two topologically equivalent torus knots/unknots with different aspect ratio may attain same energy level. 

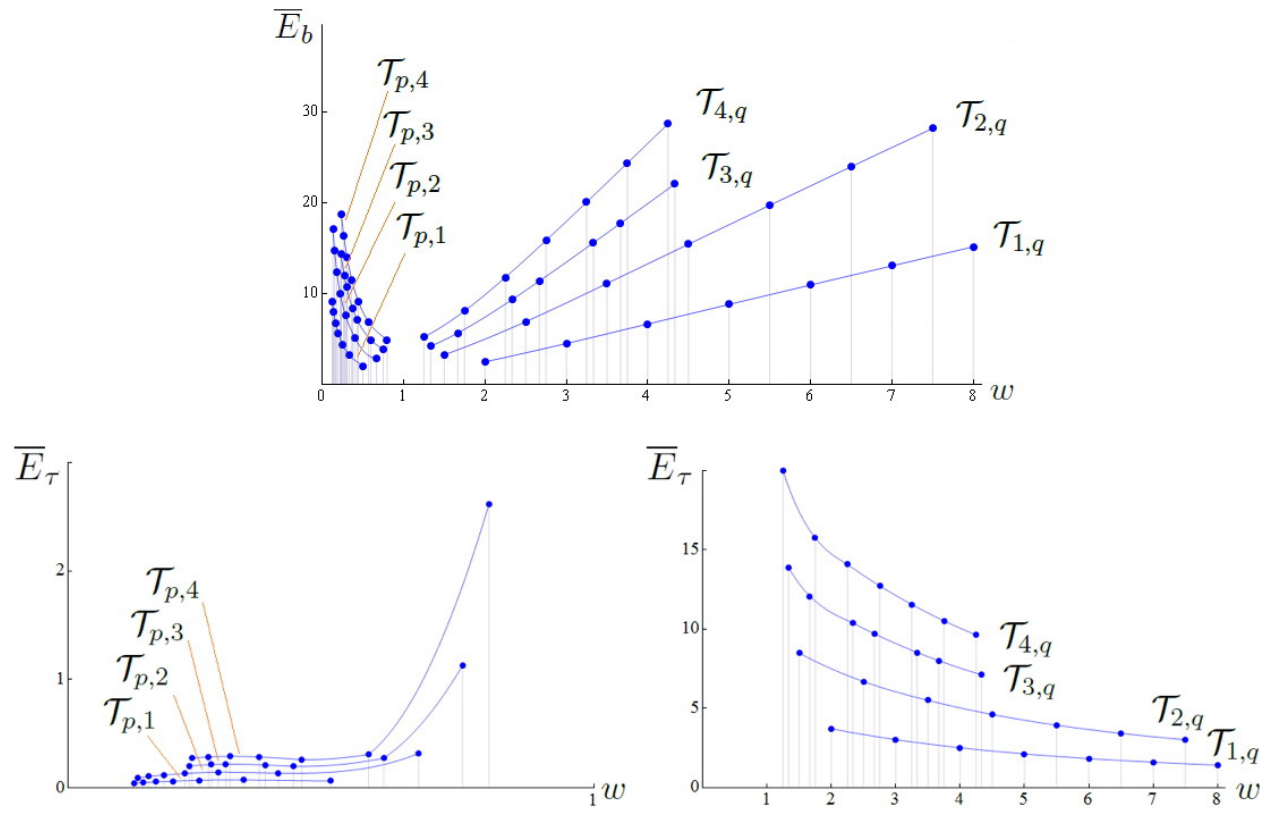

$$
\lambda=0.5
$$

Fig. 7. Nondimensional bending energy $\bar{E}_{b}$ and torsional energy $\bar{E}_{\tau}$ against winding number $w$; all knots/unknots are evaluated for $\lambda=0.5$ and $R=1$. $\mathcal{T}_{p, 1}$ and $\mathcal{T}_{1, q}, p, q=\{2,3,4,5,6,7,8\}$; $\mathcal{T}_{p, 2}$ and $\mathcal{T}_{2, q}, p, q=\{3,5,7,9,11,13,15\} ; \mathcal{T}_{p, 3}$ and $\mathcal{T}_{3, q}, p, q=\{4,5,7,8,10,11,13\} ; \mathcal{T}_{p, 4}$ and $\mathcal{T}_{4, q}$, $p, q=\{5,7,9,11,13,15,17\}$. Interpolation is made for visualization purposes.

\section{Pohl's Self-Linking Number and Călugăreanu's Invariant}

If $\mathcal{T}_{p, q}$ is endowed with framing (given by a ribbon unit vector field $\hat{\mathbf{N}}$, defined pointwise normal to the curve), the Călugăreanu-White-Fuller's theorem [23, 33, 34] (see also $[20,25]$ ) relates Pohl's geometric self-linking number $\mathrm{SL}=\mathrm{Wr}+\mathcal{T}[35]$ (where $\mathcal{T}=T / 2 \pi$ is the normalized total torsion) to the topological self-linking number Lk, by

$$
\mathrm{Lk}=\mathrm{Wr}+\mathcal{T}+\mathcal{N}=\mathrm{SL}+\mathcal{N}
$$

where the intrinsic twist $\mathcal{N}$ (an integer) denotes the number of full rotations of $\hat{\mathbf{N}}$ around the base curve of the ribbon, all along the curve. The quantity $\mathcal{T}+\mathcal{N}=\mathrm{Tw}$ is the total twist number. In absence of inflection points Pohl's self linking number $\mathrm{SL}$ is known to be an isotopy invariant of the curve. We have:

Theorem 5.1 ([19]). Let $\mathcal{T}_{p, q}$ be a torus knot/unknot. Then

$$
|\mathrm{SL}|= \begin{cases}q(p-1) & \text { if } 0<\lambda<\lambda_{\mathrm{cr}}, \\ p q & \text { if } \lambda_{\mathrm{cr}}<\lambda<1 .\end{cases}
$$


In [19] the negative values of SL are given by the parametrization associated with the handedness of $\mathcal{T}_{p, q}$. Direct computation of $\mathrm{Wr}$ and $\mathcal{T}$ for all knots/unknots considered in Sec. 4 are in good agreement with the results of Theorem 5.1.

The rôle of inflectional states is clarified by comparing the right-hand side of Eq. (5.1) to (5.2). Since $\mathrm{Lk}$ is a topological invariant of the framed $\mathcal{T}_{p, q}$, Lk remains unchanged when $\mathcal{T}_{p, q}$ goes through an inflectional configuration at $\lambda=\lambda_{\mathrm{cr}}$. As discussed in $[20]$ this produces a jump in SL given by $[\mathrm{SL}]=p q-[q(p-1)]=q$, that is the number of poloidal wraps of $\mathcal{T}_{p, q}$. Since we have

$$
\mathrm{Lk}-\mathrm{SL}=\mathcal{N}=q
$$

we can state the following:

Corollary 5.2. Let $\mathcal{T}_{p, q}$ be parametrized by Eqs. (2.3). As $\mathcal{T}_{p, q}$ goes through an inflectional state given by $\lambda=\lambda_{\mathrm{cr}}$, we have $[\mathcal{N}]=q$.

This result may find useful applications in many physical systems, and justifies the conjecture made long time ago by Moffatt and Ricca [[20], p. 426] regarding the inflectional transition of a torus unknot through a critical aspect ratio.

\section{Torus Knots/Unknots in Applications}

Geometric and topological aspects of torus knots/unknots play an important rôle in several applied contexts. Here we provide a brief review of some of these applications.

\subsection{Braided magnetic fields in the solar corona and in plasma physics}

Magnetic fields may form highly braided structures at all scales. Evidence for braiding comes from direct observation of coronal structures (see Fig. 8(a)) and photospheric motion. Plasma loops are continuously formed in the solar corona and in stellar atmospheres as gigantic arches. Since a great amount of energy eventually released to outer space is initially accumulated in these loops, it is particularly important to provide mathematical models of magnetic flux loops to understand basic properties and dynamics; this information can help to estimate energy contents and transfers between regions in space. Various attempts to relate free energy contents with structural complexity have been carried out [36].

Braiding of magnetic fields also take place inside tokamaks, where fields trapped within a toroidal vessel (Fig. 9(a)) can get highly twisted by the action of toroidal and poloidal electric currents.

By identifying the magnetic field with torus knots/unknots strands (see Fig. 8(b)) one provides an idealized setting to study the effects of geometry and topology on the dynamics and evolution of plasma loops as well as the behavior of 


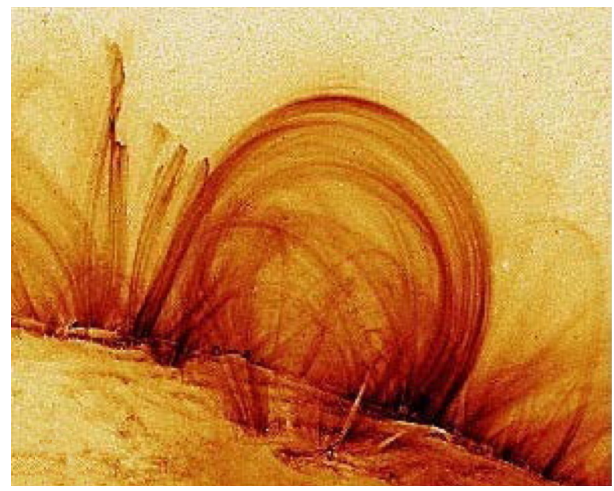

(a)

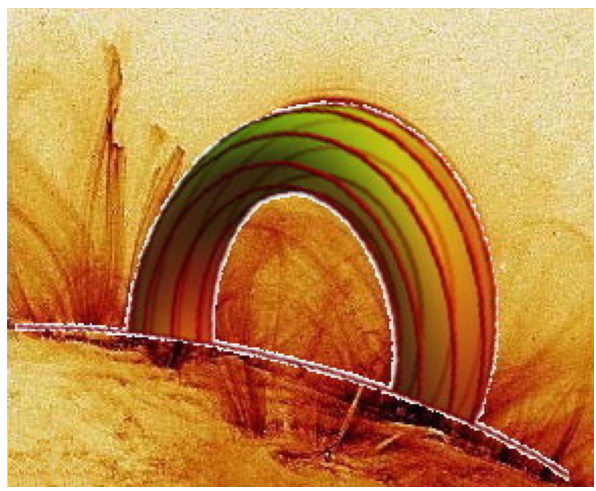

(b)

Fig. 8. (Color online) (a) Observation of a typical solar coronal loop (TRACE mission, November 6, 1999, 2 UT). (b) The same event with magnetic fields (red online) superimposed, modeled by torus knot $\mathcal{T}_{9,2}(\lambda=0.2, R=5)$.

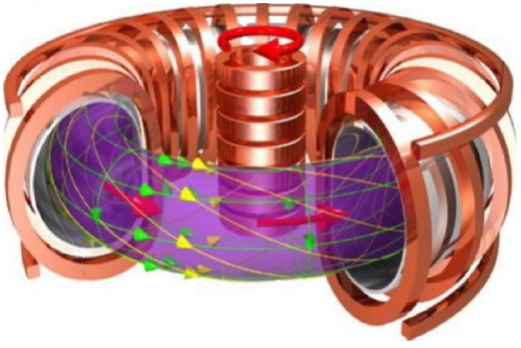

(a)

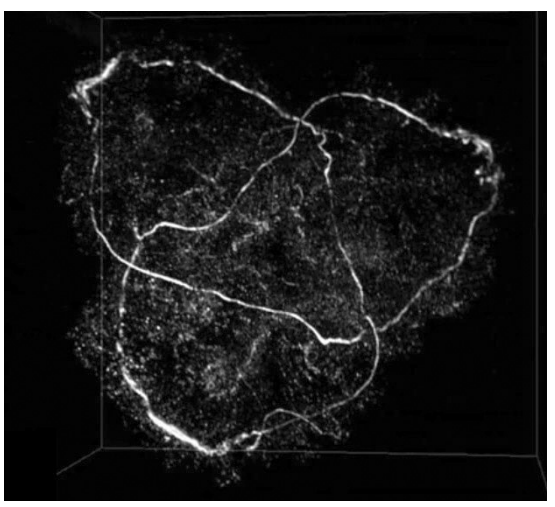

(b)

Fig. 9. (Color online) (a) Twisted magnetic field lines (yellow online) confined to the plasma region (purple online) in the Tokamak vessel. (b) A trefoil knotted vortex filament in water: the filament is visualized by the white paths formed by tiny air bubbles trapped by the region of low pressure [39].

magnetic fields in confined tokamak plasma. In both contexts inflectional configurations play an important rôle by triggering changes in twist/writhe helicity, kink instability and energy release during evolution [37].

\subsection{Vortex filaments in classical fluids, dynamical systems and quantum fluids}

Torus knot solutions to vortex filament motion have been found in the context of the so-called Localized Induction Approximation (LIA) of the Biot-Savart law for Euler's equations $[6,38]$ and studied in superfluids $[12,13]$. Real vortex torus knots 


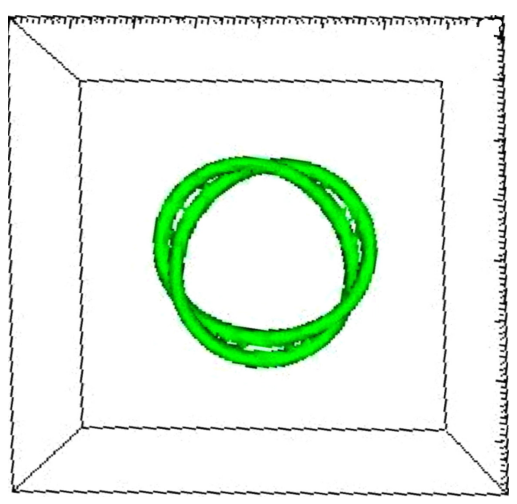

(a)

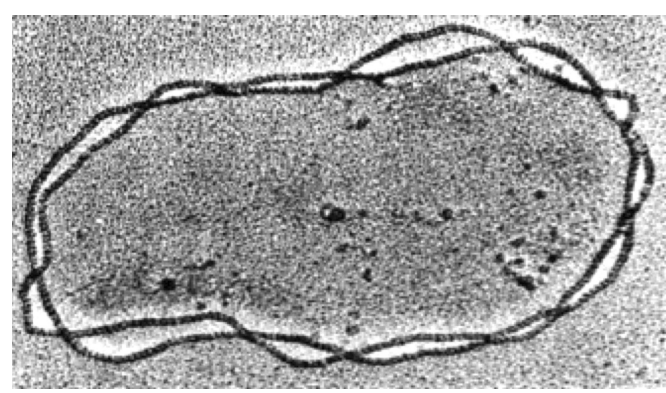

(b)

Fig. 10. (a) Trefoil knot in Bose-Einstein condensate [14]. (b) DNA torus knot $\mathcal{T}_{2,13}$ produced by topoisomerase action in laboratory experiments [42].

have been produced in water by laboratory experiments (see Fig. 9(b)) [39]. Search for torus knot solutions in dynamical systems has led to the discovery of knotted solutions in Lorenz-type of differential equations [40], as well as in the mathematical study of Euler's equations [41]. In the context of quantum fluids, defects in the shape of torus knots have been found in superfluid helium and in Bose-Einstein condensates (Fig. 10(a)) [14], and further work on the relations between topological complexity, energy and helicity is in progress. In these cases, curvature and writhe information, directly measurable from observations, have proven to be very useful to provide dynamical and energetic estimates for such systems.

\subsection{DNA macromolecules and elastic systems}

Various types of torus knots have been produced by topoisomerase actions in laboratory and computational experiments in DNA biology (Fig. 10(b)) [42, 43]. To extract information on structural and functional aspects of DNA (e.g. morphological packaging and protein coding) knot distribution has been analyzed in various systems revealing, for example, the preferential formation of torus knots in phage capsids [5]. Further work to establish probability distribution associated with the preferential formation of torus knots is in progress (Mariel Vasquez, private communication) and attracts considerable attention. Since biological knots are subject to electrostatic forces and move in a viscous fluid under the action of gravity and drag, information on the wet surface (associated with global geometric properties of ideal knots) are also useful to estimate packaging [32] and sedimentation properties in vivo.

To a first-order approximation, a DNA macromolecule can be modeled by a flexible elastic filament. Hence, a first-order, linear approximation to elastic energy is used to extract information on bending and torsional energy due to curvature and 
torsion of the filament axis [1,44]; relations with lower bound torus knot energy configurations [45] can be used to estimate energy levels of more complex knot types.

\section{Acknowledgments}

C.O. and R.L.R. would like to express their gratitude to the Centro di Ricerca Matematica Ennio De Giorgi (Pisa, Italy) and the Isaac Newton Institute for Mathematical Sciences (Cambridge, UK) for financial support and hospitality during the preparation of this work.

\section{References}

[1] B. D. Coleman and D. Swigon, Theory of self-contact in Kirchhoff rods with applications to supercoiling of knotted and unknotted DNA plasmids, Phil. Trans. R. Soc. Lond. A 362 (2004) 1281-1299.

[2] D. Kim and R. Kusner, Torus knots extremizing the Möbius energy, Exp. Math. 2 (1993) 1-9.

[3] K. W. Olsen and J. Bohr, A principle for ideal torus knots, EPL 103 (2013) 30002.

[4] J. Arsuaga, M. Vazquez, P. McGuirk, S. Trigueros, De W. Sumners and J. Roca, DNA knots reveal a chiral organization of DNA in phage capsids, PNAS USA 102 (2005) 9165-9169.

[5] D. Reith, P. Cifra, A. Stasiak and P. Virnau, Effective stiffening of DNA due to nematic ordering causes DNA molecules packed in phage capsids to preferentially form torus knots, Nucleic Acids Res. 22 (2012) 1-9.

[6] R. L. Ricca, Torus knots and polynomial invariants for a class of soliton equations, Chaos 3 (1993) 83-91 [Erratum. Chaos 5 (1995) 346].

[7] H. Kedia, I. Bialynicki-Birula, D. Peralta-Salas and W. T. M. Irvine, Tying knots in light fields, Phys. Rev. Lett. 111 (2013) 150404.

[8] W. T. M. Irvine and D. Bouwmeester, Linked and knotted beams of light, Nature Physics 4 (2008) 385203.

[9] M. R. Dennis, R. P. King, B. Jack, K. O‘Holleran and M. J. Padgett, Isolated optical vortex knots, Nature Physics 6 (2010) 118-121.

[10] K. Hikami and A. N. Kirillov, Torus knot and minimal model, Phys. Lett. B 575 (2003) 343-348.

[11] M. Kobayashi and M. Nittab, Torus knots as Hopfions, Phys. Letts. B 728 (2014) 314-318.

[12] R. L. Ricca, D. C. Samuels and C. F. Barenghi, Evolution of vortex knots, J. Fluid Mech. 391 (1999) 29-44.

[13] F. Maggioni, S. Z. Alamri, C. F. Barenghi and R. L. Ricca, Velocity, energy and helicity of vortex knots and unknots, Phys. Rev. E 82 (2010) 026309.

[14] D. Proment, M. Onorato and C. F. Barenghi, Vortex knots in a Bose-Einstein condensate, Phys. Rev. E 85 (2012) 036306.

[15] U. Tkalec, M. Ravnik, S. Čopar, S. Žumer and I. Muševič, Reconfigurable knots and links in chiral nematic colloids, Science 333 (2011) 62-65.

[16] W. S. Massey, Algebraic Topology. An Introduction (Harcourt, Brace and World, New York, 1967). 
[17] K. Murasugi, On the braid index of alternating links, Trans. Amer. Math. Soc. 326 (1991) 237-260.

[18] T. J. Willmore, An Introduction to Differential Geometry (Oxford University Press, Oxford, 1959).

[19] E. J. Fuller Jr., The Geometric and Topological Structure of Holonomic Knots, Phd Thesis (U. Georgia, Athens, 1999).

[20] H. K. Moffatt and R. L. Ricca, Helicity and the Călugăreanu invariant, Proc. R. Soc. A 439 (1992) 411-429.

[21] R. L. Ricca and H. K. Moffatt, The helicity of a knotted vortex filament, in Topological Aspects of the Dynamics of Fluids and Plasmas, ed. H. K. Moffatt et al. (Kluwer, Dordrecht, The Netherlands, 1992), pp. 225-236.

[22] S. I. Rodrigues Costa, On closed twisted curves, Proc. Am. Math. Soc. 109 (1990) 205-214.

[23] F. B. Fuller, The writhing number of a space curve, PNAS USA 68 (1971) 815-819.

[24] F. B. Fuller, Decomposition of the linking number of a closed ribbon: A problem from molecular biology, PNAS USA 75 (1978) 3557-3561.

[25] M. R. Dennis and J. H. Hannay, Geometry of Călugăreanu's theorem, Proc. R. Soc. A 461 (2005) 3245-3254.

[26] J. Aldinger, I. Klapper and M. Tabor, Formulae for the calculation and estimation of writhe, J. Knot Theory Ramifications 4 (1995) 343-372.

[27] I. Fàry, Sur la courbure totale d'une courbe gauche faisant un nœud, Bull. Soc. Math. France 77 (1949) 128-138.

[28] J. Milnor, On total curvatures of closed space curves, Math. Scan. 1 (1953) 289-296.

[29] H. von der Mosel, Elastic knots in Euclidean 3-space, Ann. Inst. H. Poincaré 16 (1999) 137-166.

[30] C. Adams, R. Hudson, R. Morrison, W. George, L. Starkston, S. Taylor and O. Turanova, The spiral index of knots, Math. Proc. Camb. Phil. Soc. 149 (2010) 297315.

[31] A. E. H. Love, A Treatise on the Mathematical Theory of Elasticity (Cambridge University Press, Cambridge, 1920).

[32] R. L. Ricca and F. Maggioni, Multiple folding and packing in DNA modeling, Comp. Math. Appl. 55 (2008) 1044-1053.

[33] G. Călugăreanu, Sur les classes d'isotopie des nœeds tridimensionnels et leurs invariants, Czechoslovak Math. J. 11 (1961) 588-625.

[34] J. H. White, Self-linking and the Gauss integral in higher dimensions, Am. J. Math. 91 (1969) 693-728.

[35] W. F. Pohl, The self-linking number of a closed space curve, J. Math Mech. 17 (1968) 975-985.

[36] M. A. Berger, Energy-crossing number relations for braided magnetic fields, Phys. Rev. Lett. 70 (1993) 705-708.

[37] R. L. Ricca, Inflectional disequilibrium of magnetic flux tubes, Fluid Dyn. Res. 36 (2005) 319-332.

[38] S. Kida, A vortex filament moving without change of form, J. Fluid Mech. 112 (1981) 397-409.

[39] D. Kleckner and W. T. M. Irvine, Creation and dynamics of knotted vortices, Nat. Phys. 9 (2013) 253-258.

[40] J. S. Birman and R. F. Williams, Knotted periodic orbits in dynamical systems I: Lorenz's equations, Topology 22 (1983) 47-82. 
[41] A. Enciso and D. Peralta-Salas, Knots and links in steady solutions of the Euler equation, Ann. Math. 175 (2012) 345-367.

[42] S. J. Spengler, A. Stasiak, N. R. Cozzarelli, The stereostructure of knots and catenanes produced by phage $\lambda$ integrative recombination: Implications for mechanism and DNA structure, Cell 42 (1985) 325-334.

[43] K. Shimokawa, K. Ishihara, I. Grainge, D. J. Sherratt and M. Vazquez, FtsKdependent XerCD-dif recombination unlinks replication catenanes in a stepwise manner, PNAS USA 110 (2013) 20906-20911.

[44] R. L. Ricca, The energy spectrum of a twisted flexible string under elastic relaxation, J. Phys. A: Math. and Gen. 28 (1995) 2335-2352.

[45] H. Gerlach, P. Reiter and H. von der Mosel, The elastic trefoil is the twice covered circle, arXiv:1510.06171 [math.DG] 21 Oct., 2015 